\title{
A REVIEW OF MODELLING APPROACHES ON TIDAL ANALYSIS AND PREDICTION
}

\author{
Auwal Garba Abubakar ${ }^{1,2}$, Mohd Razali Mahmud ${ }^{1}$ Kelvin Kang Wee Tang ${ }^{1}$, \\ Alhaji Hussaini ${ }^{3}$. Nur Hidayah Md Yusuf ${ }^{1}$ \\ ${ }^{1}$ Faculty of Built Environment and Surveying, Universiti Teknologi Malaysia, 81310 Johor Bahru, Malaysia \\ ${ }^{2}$ Department of Surveying and Geo-Informatics, College of Environmental Studies, Waziri Umaru Federal Polytechnic Birnin \\ Kebbi, Kebbi State, Nigeria. \\ ${ }^{3}$ Department of Geography, Aminu Saleh College of Education Azare, Bauchi, Nigeria. \\ gaauwal2@live.utm.my
}

KEY WORDS: Tides, Harmonic Analysis, Tidal Prediction, Hybrid Model.

\begin{abstract}
:
Tide height depends on both long-term astronomical effects that are principally affected by the moon and sun and short-term meteorological effects caused by severe weather conditions which are very important tasks for human activities, safe marine navigation in shallow areas, oceans and coastal engineering work. Conventional tidal forecasting techniques are based on harmonic analysis, which is a superposition of many sinusoidal constituents with three parameters amplitudes, Phase and frequencies using the least squares method to determine the harmonic parameters. However, harmonic analysis required a large number of parameters and long-term tidal measured for precise tidal level predictions. Furthermore, what seems to stand out by the other researchers on traditional harmonic methods, was its limitation when short data are involved and rely on based on the analysis of astronomical components and they can be insufficient when the influence of non-astronomical components such as the weather, is important. Therefore, conventional harmonic analysis alone does not adequately predict the coastal water level variation, in order to deal with these situations and provide predictions with the desired accuracy, with respect to the length of the available tidal record, an alternative approach has been developed by various tidalist. In this study the state - of - art for tidal analysis and prediction techniques that have proven to be successful in a variety of circumstances have been reviewed in a systematic and consistent way for holistic understanding with a view to provide a reference for future work, showing their main mathematical concepts, model capabilities for tidal analysis and prediction with their limitations.
\end{abstract}

\section{INTRODUCTION}

Tides are alternating variation of the sea level as influence mainly by astronomical component such as gravitational forces exerted by the moon, sun and the rotation of the earth (Doodson, 1954; Godin, 1972; Shum et al., 2001; Pugh and Woodworth, 2014; Haigh, 2017). However, other factors also reflect non-astronomical sources such as the wind, weather, seafloor topography and local water depth (Devlin, 2016; West et al., 2016). The Newton gravitational theory gives a wider understanding that leads to the discovery and understanding of the tidal phenomena. Therefore, tides are the periodic phenomena that can be express as the superposition of sinusoidal function each function has three parameters, namely frequency, amplitude and phase (Cai et al., 2018).

Coastal waterways play a critical economic role and affect shipping, oil and natural gas production, recreation tourism, fisheries and environmental habitat. Precise knowledge and understanding of tidal and subtidal water level fluctuation and circulation along the coastal water is important for safe marine navigation, water quality emergency management such as oil spill response, search and rescue operation and evacuation during extreme weather events. Due to speedy development on the coastal infrastructural development and coastal engineering projects recently in the world, there is a need for precise information on site-specific water level height. Therefore, it's quite obvious to have a reliable water level prediction tool that can cater the need for coastal engineering port and harbour activities.
Over the years a series of methodology for tidal analysis and prediction to ameliorate the accuracy of water level prediction have been established among the most popular one is the classical harmonic analysis which was established by Kelvin and improved by Darwin, Doodson, and others. This study tends to further examine and review up to date coverage of tidal analysis and prediction modelling techniques that have demonstrated to be successful in a variety of circumstances with a view to providing a broad description of the existing state-ofthe art in the field. A systematic and consistent way was adopted to review the classical as well as the novel methods through a number of web-based articles from relevant databases, such as Web of Science, Scopus, ScienceDirect, IEEE Xplore Digital Library, and Elsevier were accessed through search engines, which lead to the eventual selection of 50 tidal analysis and prediction modelling research articles that were published.

The present paper is organized as follows. The literature on tidal analysis and forecasting models' approaches are presented in Section 2. Summary of the strengths/limitations of the previous researches were discussed in Section 3. Conclusion and future direction of tidal analysis and prediction are coveredup in Section 4.

\section{AN OVERVIEW OF TIDAL ANALYSIS AND PREDICTION MODELLING TECHNIQUES}

There have been numerous advances in tidal analysis and prediction (Imani et al., 2018), a standout amongst the best and 
generally utilized methodologies has been and continue to be, the classical harmonic analysis where in the energy at explicit tidal frequencies is dictated by a scientific fitting system, typically least squares (Zhang et al., 2017). However, this review, focus just on tidal analysis and prediction modelling approaches from the early stage of a single technique to present time of hybridization. An early clarification of tide can be traced back to 1616 when Galileo Galilei ascribed the tide to water splashing due to the earth's movement around the sun, at the same time Johannes Kepler made some suggested based upon ancient observation and correlation that the moon caused the tide (site), Isaac Newton (1642-1727) was the first person to explain tides by developing his universal law of gravitation and equilibrium tidal theory. Isaac Newton used his theory of universal gravitation to establish the tide-generating forces as due to the moon and sun attractions to the rotating earth. PierreSimon Laplace (1749-1827) established the dynamical theory known as equilibrium theory of tide based on Newton's work, which pronounced the ocean's real response to tidal forces.

The Laplace tidal equations are still in use today William Thomson (1824-1907) developed the concept of harmonic analysis by application of Fourier analysis to the tidal motions. A concept which was further developed and extended by George Darwin: Darwin's (1845-1912) Darwin can be traced back as the funder of classical tidal harmonic analysis which was further refined by Doodson (1972). Doodson recognized 388 different tidal frequencies and formulated a practical system for specifying the different harmonic components of the tidegenerating potential, the Doodson Numbers, a system still being used today to signify the different tidal species and constituents. Since the mid-twentieth century further analysis has created a lot a greater number of terms than Doodson's 388. Around 62 constituents are of adequate size to be considered for conceivable use in marine tide prediction, yet in some cases a lot less even than that can predict tides with improved precision. Table 1 shows the major tidal constituents contributing to the astronomical tide

Table 1. Major Harmonic Constituent

\begin{tabular}{|l|l|l|}
\hline Symbol & Constituent name & Period (T) \\
\hline $\mathrm{M}_{2}$ & Principal lunar semidiurnal & 12.42 \\
$\mathrm{~N} 2$ & Principal solar, semidiurnal & 12.00 \\
$\mathrm{~K} 2$ & $\begin{array}{l}\text { Elliptical lunar, semidiurnal } \\
\text { Declinational luni-solar, } \\
\text { semidiurnal }\end{array}$ & 12.66 \\
$\mathrm{~K} 1$ & $\begin{array}{l}\text { Declinational luni-solar, } \\
\text { diurnal } \\
\text { Principal lunar, diurnal } \\
\text { O1 }\end{array}$ & 23.93 \\
$\mathrm{Sa}$ & Meteorological, annual & Year \\
\hline
\end{tabular}

Continuously other tide analysis and prediction techniques based on Harmonic analysis were largely proposed in the literature. The most relevant is the work of (Guo et al., 2018) to capture the slow variation of tidal amplitudes and phases, based on variations on a timescale of multiple months or longer, using an empirical Harmonic Analysis Model. Harmonic analysis of tidal and sea-level data was implemented by. (Stephenson, 2017) to estimate up to 409 different harmonic tidal constituents, considering daily nodal corrections in Australia. (Badejo and Akintoye, 2017) apply the least squares tidal harmonic analysis to determine harmonic constants and were substituted into a tidal prediction model to predict hourly tidal data and tidal predictions at 5 minutes' intervals at Lagos harbour Nigeria. The University of Hawaii Sea Level Center in collaboration with the National Oceanographic Data Center (NODC) developed a tidal analysis and prediction tool based on harmonic tidal analysis using linear least squares named "The Sea Level Processing Package" (SLPR2), the tide prediction algorithm of SLPR2 uses a maximum of 68 harmonic constituents which provides reliable sea level information estimate of a specific location.

The predictive capability of SLPR2 for a one-year period was investigated by (Rose and Bhaskaran, 2015) in the head Bay of Bengal region located along the east coast of India. And the results from SLPR2 indicate considerable correlation between predicted tide with the measured tide. (Foreman et al., 2009) develop and test a more versatile harmonic analysis technique that can accept randomly sampled data and embed the nodal and astronomical argument corrections and multiple inference calculations into an overdetermined matrix that is solved using singular value decomposition (SVD) technique. Many other researchers such as (Amiri et al., 2014; Najibi, et al., 2013; Mousavian and Hossainali, 2012; Ew and Ugust, 2003; Pawlowicz et al., 2002; Jay and Kukulka, 2003) apply harmonic analysis technique.

Tidal analysis and prediction technique based on Kalman filtering were largely proposed in the literature. The most recent is the work of (Slobbe et al., 2018) apply the Kalman filter approach to combine a hydrodynamic model to derive lowest astronomical tide (LAT) surface with tide gauge records in the Dutch North Sea and Wadden Sea, by using the Kalman filter approach Slobbe was able to obtained a smooth reference surface of the Dutch Wadden Sea that is LAT at the North Sea boundary within a few centimetres. (Okwuashi and Olayinka, 2017) make use of the Kalman Filter. Okwuashi based his work on seven harmonic constituents: M2, S2, N2, K2, K1, O1 and $\mathrm{P} 1$, to compute tidal form factor $\mathrm{F}=0.1955$ which shows a good indication that the tide is semi-diurnal, the result of the experiment can be trusted, since Nigerian coastal waters are characteristically semi-diurnal in nature. Sea level prediction based on harmonic model with their parameter estimation by the Kalman filter algorithm was tested for Macau by (Choi et al., 2000).

A Kalman filter method was used for tidal analysis and prediction using a short length data (a few days) of tide measurements to predict tide levels over a longer time by (Pei $e t$ $a l ., 1996)$ the result of the tide levels predicted by the Kalman filter approach method was in good agreement with the observed data at the Kaohsiung Harbour, Taiwan. A tide gauge data from six selected islands in the tropical Pacific to predict sea level height using Kalman filter was tested, by (Miller and Cane, 1989) The error model is a simple covariance function with parameters fit from the observed differences between the tide gauge data and the model out output. The calibrated error model is used in a Kalman filtering scheme to generate monthly sea level height anomaly maps. The results were quite encouraging.

Harmonic techniques have been discussed in this literature, but its accuracy depends entirely on long-term tidal record data. This is the major shortcoming of the harmonic technique. However, tidalist applied the Kalman filter model to determine 
harmonic parameters with a limited data. However, the Kalman Filter model is only relevant for the short time prediction.

Other technique such as artificial neural network, back propagation neural network and wavelet transformation were proposed in the literature. (Tsai and Lee, 1999a) was first to apply Neural network for tide forecasting by utilizing the field data of both diurnal and semidiurnal tides. However, their model was applied for the forecast of diurnal and semi-diurnal tides, mixed tides are bound to happen in the field than diurnal and semi-diurnal tides. As the need for accurate ocean tide models has become increasingly important over the recent years, an application of the back-propagation neural network using short-term measurement data for long-term tidal predictions was presented by (Lee, 2004) at Taichung Harbor in Taiwan. Back-propagation neural network technique proficiently predicts the long-term tidal levels when comparison was made with a conventional harmonic technique. Tide gauge data collected at the town of Burgas, located at the western Bulgarian Black Sea coast for the period seven years (19902003) were used as input data to carry out a several tests of different Artificial Neural Network (ANN) architectures and learning algorithms to assess their applicability as competitive methods to the harmonic analysis by (Pashova and Popova, 2011).

Their founding indicate that the artificial neural technique is appropriate for short and long-term forecasts of the sea level prediction and sea level parameter identification. (Lee and Lee, 2014) presents a model of coastal high and low water prediction using feed forward back-propagation (FFBP) neural network and generalized regression neural network (GRNN), to coastal high and low water level prediction. Lee made use of 30-days of hourly tidal and five main tidal constituents to obtain a good result. However, prediction for one-month and one-year period indicates that the network models are unable to predict the high and low water accurately. The results for one-month prediction provide some level of effective prediction, but for a one-year simulation the result has much larger errors. Tidalist such as (Makarynska and Makarynskyy, 2008), (Meena and Agrawal, 2015), (Deo and Chaudhari, 1998), (Muhammad et al., 2015) all made the utilization of Artificial Neural Network (ANN) model for tidal analysis using the short-time water level data as an alternative to conventional harmonic analysis method. Previous study shows that Artificial Neural Network (ANN) is found to be an efficient technique in forecasting the tidal data time series for a short duration, however, the ANN model remain site specific and did not ameliorate the accuracy of the predicted tide when long-time prediction is required with a short data.

To understand the numerous divergence of tidal phenomena from an assumed statistical stationarity or exact periodicity inherent in traditional harmonic and other tidal methods, (Flinchem and Jay, 2000) introduces Wavelet Transform for Tidal Analysis Methods "Use of wavelets allows determination of the degree of non-stationarity present in time series, such as estuarine and shelf currents, usually treated as stationary. Wavelets also provide a consistent analysis of tidal and nontidal variance, a feature often essential for dynamical analyses of non-stationary tides"(Flinchem and Jay, 2000). Wavelet analysis provides a consistent, linear analysis of tidal and nontidal variance and reveals features that harmonic analysis on a Fourier transform approach could not elucidate. (El-Diasty and Al-Harbi, 2015), proposed and developed highly nonlinear and efficient wavelet network model for accurate water levels modelling and prediction using short water level measurements with meteorological effects. The performance accuracy of the developed wavelet network model shows that, the differences between water level measurements and the modelled predicted values, fall within the $-5 \mathrm{~cm}$ and $+5 \mathrm{~cm}$ range and root-meansquared (RMS) errors fall within $1-6 \mathrm{~cm}$ range. A new method named "Inaction method" (also referred to as a "line-pass" filter, in the filtering language) for predicting short-term tidal levels was recently introduced by (Cai, and Wang, 2018). The "Inaction method", is based on normal time frequency transform (NTFT) which is another name for normal morlet wavelet transform, can recognize and extract harmonic/quasi harmonic components of a tidal signal without an inverse transform. Tide gauge measurements from Quarry Bay, Hong Kong was tested and the results demonstrate the capacity of the "Inaction method" to generate a short-term tidal level forecast based on correlation coefficient (0.92-0.99), root mean square error (about $4 \%-8 \%$ of the tidal range). However, the results also show that the prediction was worse as the lead time increases. So, "Inaction method" is only appropriate for the short-term tidal level predictions.

Tidal analysis composed of astronomical tide parts caused by celestial bodies' movement and the non-astronomical tide parts caused by various meteorological and other environmental factors, due to several features that differentiate tidal analysis from most other geophysical applications of time-series analysis resulting to the disturbance factors of tidal level which are very intricate. To ameliorate the accuracy of tidal analysis and prediction tidal researchers have shifted their research work toward hybrid modelling which is the current-state of -the- art The most recent and up to date is the work of (Li et al., 2019) In action method (IM) is combined with lease square estimation (LSE) to predict long-term tidal levels by Sida Li.

The results showed that, the tidal level predictions using the LSEIM were enhanced in accuracy by $9-15 \mathrm{~mm}$ with a lead time of two months, with respect to those using purely LSE. Water level height was inferred by making the first-order difference with the raw datasets obtain at Anqing-Wuhu-Nanjing of the Yangtze River by (Zhong et al., 2019), Zhong developed a hybrid model of an artificial neural network with Kalman filter for water level forecasting. The Hybrid model predictions were satisfactory. Aside from, some discrepancies during the flood and dry seasons when the field observations show severe variations. (Liu, and Zhu, 2019) proposed a combined tidal forecasting model based on harmonic analysis and autoregressive integrated moving average support vector regression (ARIMA-SVR). Liu proposed hybrid model to ameliorate the low precision associated with single prediction models.

However, harmonic analysis as a part of the combined model has the problem of the high cost of obtaining large amounts of data from on-site monitoring equipment. (Wang and Yuan, 2018) explore a more advanced method for forecasting tide levels of storm surge caused by typhoons, on the basis of successive five-year tide level and typhoon data at Luchaogang, China, a BP neural network Hybrid model was developed by Wang using six parameters of typhoons as input parameters and the relevant tide level data as output parameters, their results have shown that the hybrid approach has higher accuracy in forecasting tidal level of storm surge caused by typhoons. (Eldiasty et al., 2018). Developed a hybrid harmonic analysis and wavelet network (HA-and-WN) model for accurate sea water level prediction. 
To ameliorate the general accuracy of tidal prediction and improve the low accuracy of a single harmonic analysis, (Imani et al., 2018) used another hybrid approach called extreme learning machine and relevance vector machine for sea level prediction at chiayi coast, Taiwan. A hybrid prediction mechanism was constructed by taking both advantages of harmonic analysis and neural network by (Yin, et al., 2015). (Vanlede et al., 2014) predicted tide at Scheldt (Belgium) using a combination of harmonic tidal prediction with 1D hydraulic modelling at Prosperpolder (a measurement station close to the Dutch-Belgian border).

Vanlede uses harmonic tidal prediction at a station downstream and 1D hydraulic modelling for the stations further upstream to predict the vertical tide in the Sea Scheldt (Belgium), their result shows, that the accuracy of the hybrid model however is better than the difference that can be expected between the harmonically predicted tide and the observed tide due to meteorological effects. Beside that the accuracy at downstream is naturally limited by the accuracy of the tidal analysis while the accuracy at the upstream is further limited by the accuracy of the hydraulic model used. (Karimi et al., 2013) presented a predicted hourly sea level for Darwin Harbor, Australia using two different, data driven techniques, Adaptive Neuro Fuzzy Inference System (ANFIS) and Artificial Neural Network (ANN).

\subsection{Harmonic Analysis Model Approach}

mathematically the tidal amplitudes and phase lags needed for accurate tidal predictions are determined via conventional tidal harmonic analysis approach of sea level records spanning a year or more originally propose by Godin (1972). The tidal height $h$ at any time $t$ can then be predicted from the superposition of the sinusoidal tidal constituent amplitudes $\left(\mathrm{A}_{\mathrm{k}}\right)$ and phase lags $\left(\mathrm{g}_{\mathrm{k}}\right)$, along with their astronomical arguments $\left(\mathrm{V}_{\mathrm{k}}\right)$, nodal factors $\left(\mathrm{f}_{\mathrm{k}}\right)$ and nodal angles $\left(\mathrm{u}_{\mathrm{k}}\right)$, as given by

$$
h\left(t_{j}\right)=Z_{0}+\sum_{k=1}^{n} f_{k}\left(t_{0}\right) A_{k} \cos \left[\omega_{k}\left(t_{j}-t_{0}\right)+V_{k}\left(t_{0}\right)+u_{k}\left(t_{0}\right)-g_{k}\right]+R\left(t_{j}\right)
$$

Where $\quad h\left(t_{j}\right)$ is the tidal height measurement at time $t$, $\mathrm{Z}_{\mathrm{o}}$ is the mean sea level,

$\mathrm{f}_{\mathrm{k}}\left(\mathrm{t}_{0}\right)$ and $\mathrm{u}_{\mathrm{k}}\left(\mathrm{t}_{0}\right)$ are the nodal correction modulation factors to amplitude and phase correction,

$\mathrm{V}_{\mathrm{k}}\left(\mathrm{t}_{0}\right)$ is the astronomical argument at initial epoch $\mathrm{t}_{0}(12: 00 \mathrm{UT}$ Dec 31, 1899).

$\mathrm{A}_{\mathrm{k}}$ and $\omega_{\mathrm{k}}$ are the amplitude and angular speed of constituent, respectively.

$\mathrm{g}_{\mathrm{k}}$ is the phase lag which is relative to the

Equilibrium Tide at Greenwich.

The subscript $\mathrm{k}$ represents the $\mathrm{kth}$ tidal constituent. The amplitude $A_{k}$ and phase angle gk are the so-called harmonic constants.(Godin, 1972; Foreman and Henry, 1989).

\subsection{Mathematical Concept of Kalman Filter model}

The ideologies of the Kalman filter is based on an optimal recursive estimate intended for data processing that uses indirect, inaccurate and uncertain observations to infer the parameters of interest (Seshadri, 2016, Peter, 1979). The KF algorithm which is a powerful filter basically estimates the current state of a dynamic system from incomplete noisy indirect measurements (Persson and Sharf, 2012). The KF can be applied to both linear and nonlinear processes/systems (Bezručka, 2011). The Kalman Filter consists of a measurement equation; transition equation; prediction equation and the updating equation (Choi, Mok and Tam, 2000) as follows

$$
x_{t}=F_{t} x_{t-1}+B_{t} u_{t}+w_{t}
$$

Where $\quad x_{t}$ is the state vector containing the terms required for the system at a time $\mathrm{t}$

$\mathrm{U}_{\mathrm{t}}$ is the control input vector

$F_{t}$ is the state transition matrix, responsible for applying the effect of each previous system state parameter on to the current state.

$\mathrm{B}_{\mathrm{r}}$ is the control input matrix, responsible for applying the effect of control input parameter on the control parameter $U_{t}$

$\mathrm{W}_{\mathrm{r}}$ is the Vector containing the process noise terms.

Two stages are involved in the Kalman filter algorithm they are: Time update (prediction) and measurement update (correction). The Kalman filter equations for the prediction stage are given by

$$
\begin{gathered}
{ }_{t \mid t-1}^{x_{t-1}}=F_{t-1} x_{t-1} F_{t}^{T}+B_{t} u_{t} \\
P_{t \mid t-1}=F_{t} P_{t-1 \mid t-1} F_{t}^{T}+Q_{t}
\end{gathered}
$$

The measurement update equations are given by

$$
\begin{gathered}
x_{t \mid t-1}=x_{t \mid t-1}+K_{t}\left(z_{t}-H_{t} x_{t \mid t-1}\right) \\
P_{t \mid t}=P_{t \mid t-1}-K_{t} H_{t} P_{t \mid t-1} \\
K_{t}=P_{t \mid t-1} H_{t}^{T}\left(H_{t} P_{t \mid t-1} H_{t}^{T}+R_{t}\right)^{-1}
\end{gathered}
$$

Where $\quad \mathrm{x}$ is the Estimate State.

$F$ is the State transition matrix.

$\mathrm{U}$ is the Control Variable.

$\mathrm{B}$ is the Control input matrix.

$\mathrm{P}$ is the State Variance matrix.

$\mathrm{Q}$ is the Processing noise covariance matrix.

$\mathrm{Z}$ is the Measurement Variable.

$\mathrm{H}$ is the Measurement matrix.

$\mathrm{K}$ is the Kalman Gain.

$\mathrm{R}$ is the Measurement Noise covariance matrix.

The subscript $t / t$ denote the current time period, while $t-1 / t-1$ denotes the previous time period, finally $t / t-1$ refers to the intermediate steps. 


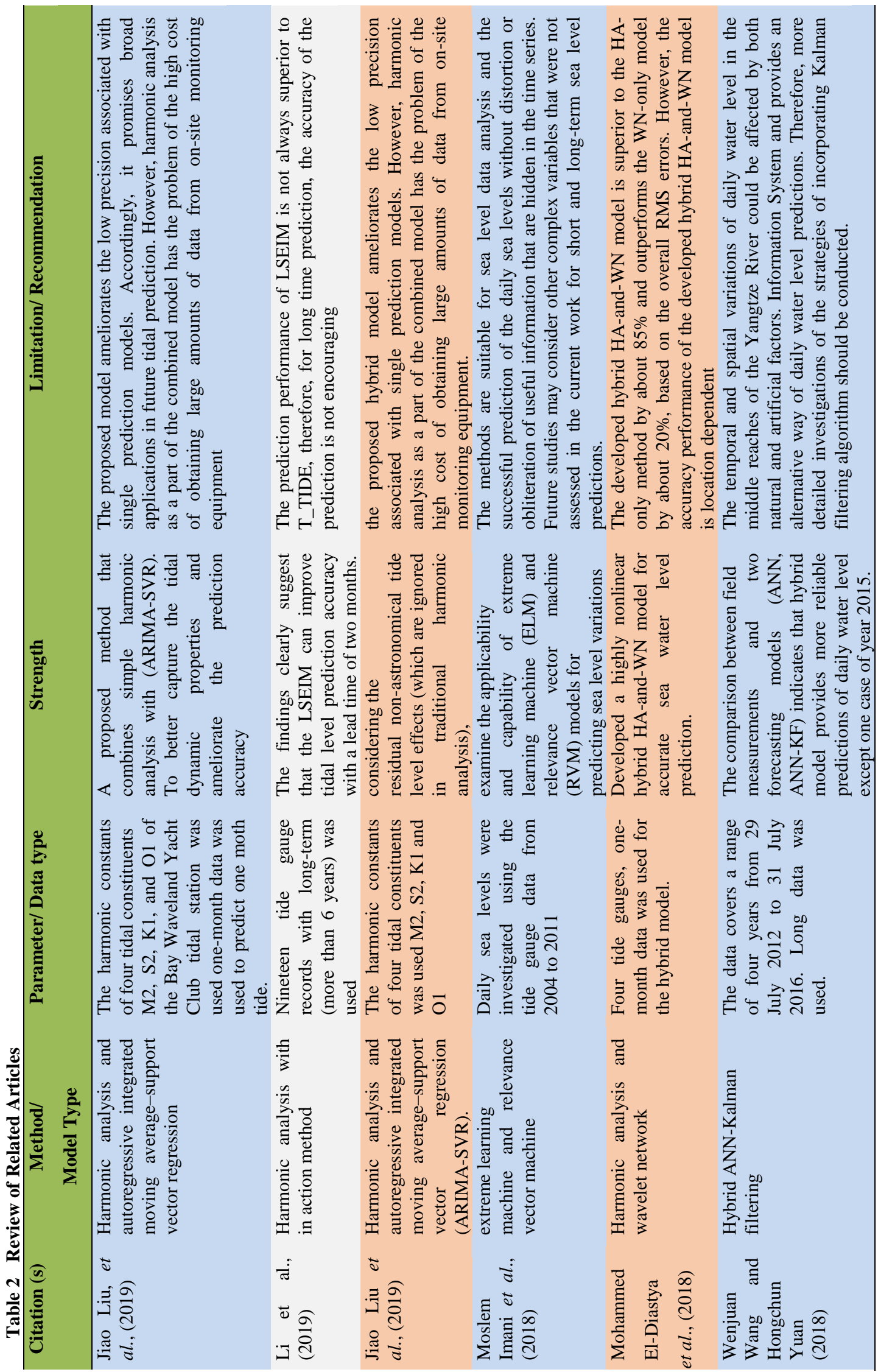




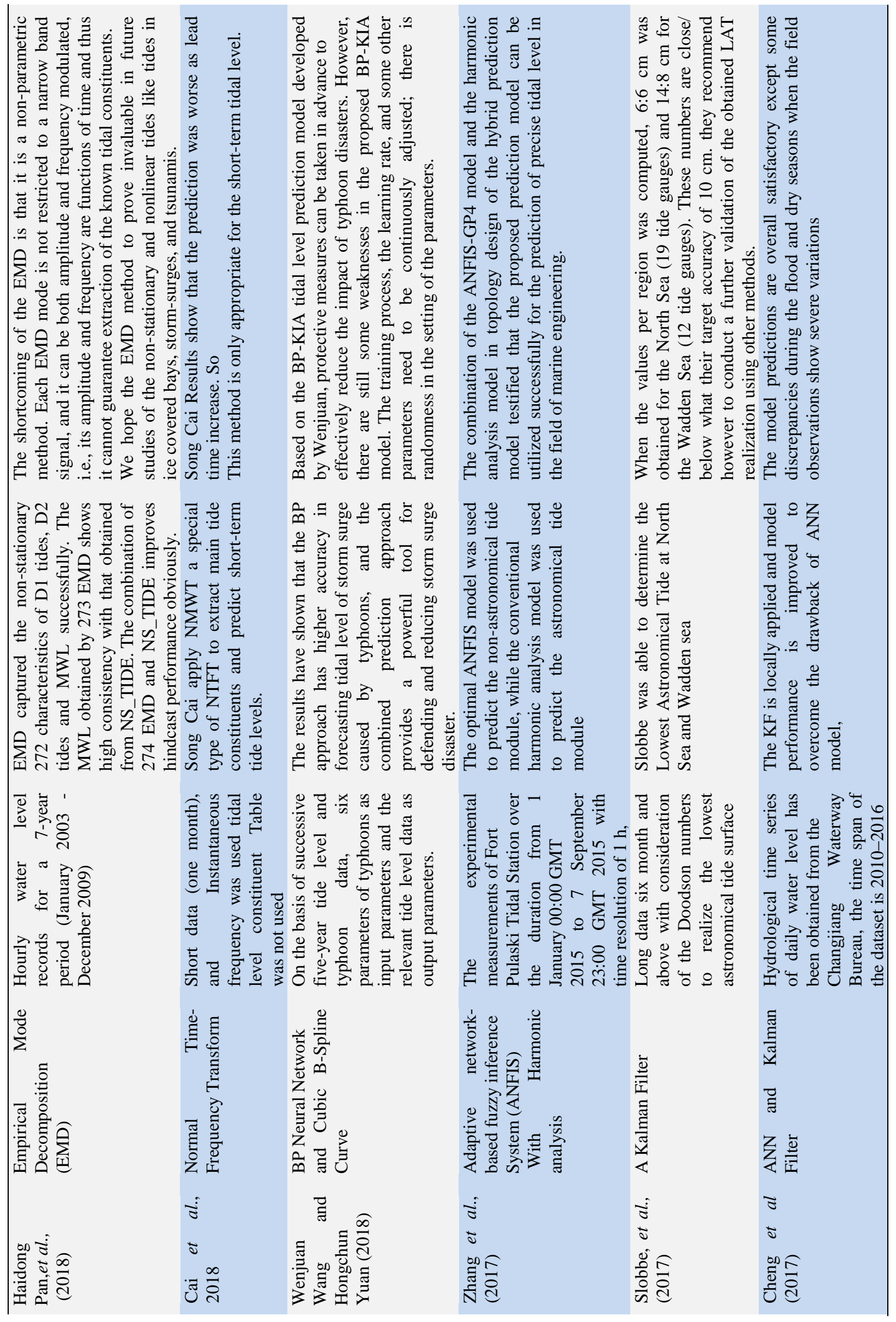




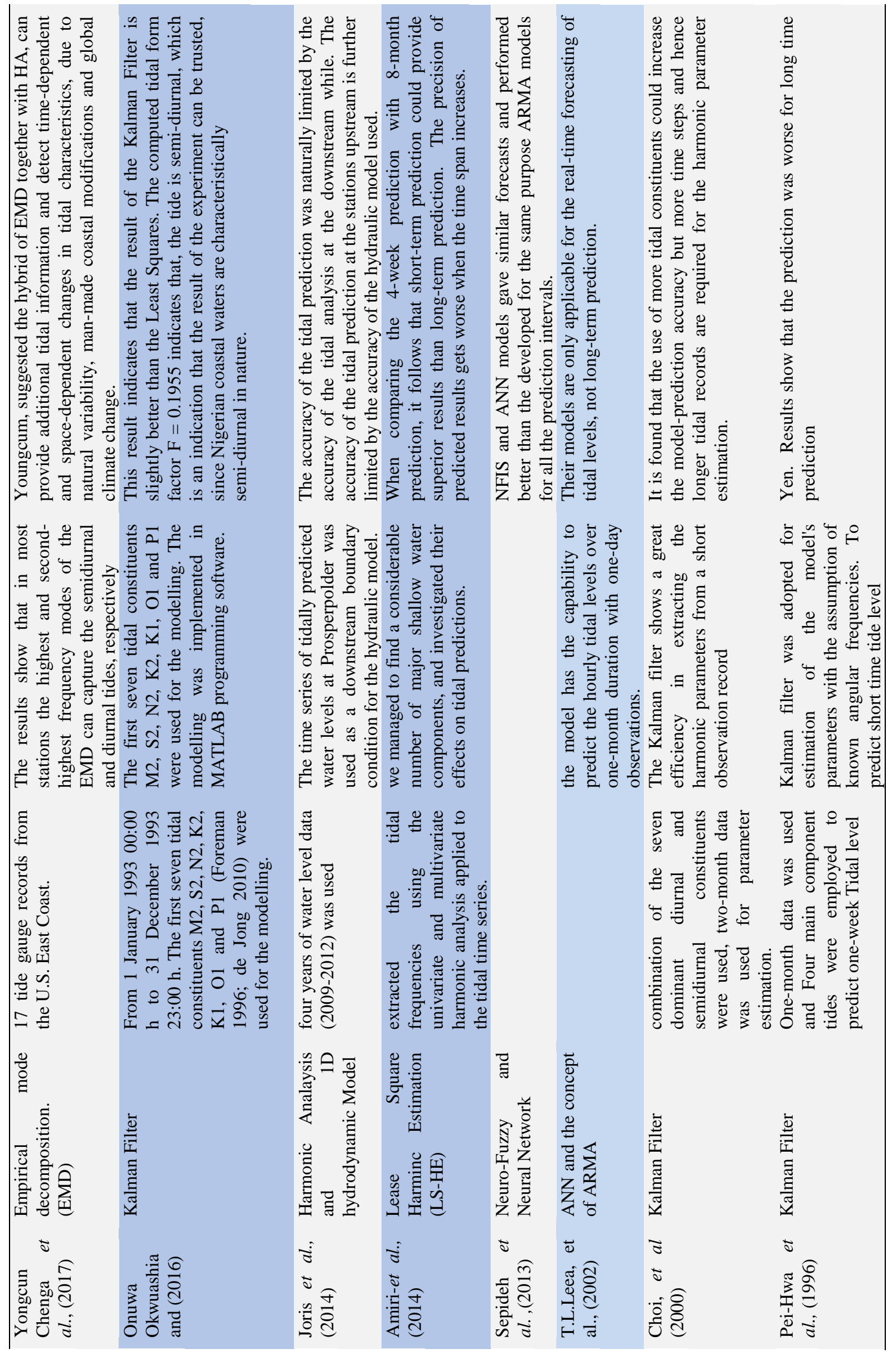




\subsection{The General concept of Artificial Neural Networks Model}

The origin of neural network was the bionomics. The natural cerebrum comprises of billions of very interconnected neurons forming a neural network. Human data processing relies upon this connectionist arrangement of sensory cell. Based on the advantage of this data processing, neural networks can easily exploit the enormous parallel local processing and distribute storage properties in the brain. The ability of biological neural networks can be modeled generally through a mathematical system called ANN by interconnecting a considerable lot of the basic neurons (Tsai and Lee, 1999b). Therefore, inputs from a multiple or single source are accepted by the neuron (Huang et al., 2003), hence produces outputs by simple processing with a predetermined non-linear function (Filippo et al., 2012) as shown in Figure1. The primary characteristics of ANN can be presented as (Recupero et al., 2013; Rumelhart, et al., 1988)

a) The ability to learn;

b) Distributed memory;

c) Fault tolerance;

d) Parallel operation.

The principle of ANNs has been well documented in the literature (Fausett, 1994; Bishop, 1995) only a brief outlook is given in this section. Neural Network consist of a typical threelayered network with an input layer (I), a hidden layer $(\mathrm{H})$ and an output layer $(\mathrm{O})$ as shown in figure2, each layer consists of several neurons and the layers are interconnected by sets of correlation weights (Consoli et al., 2014; Lee, 2002). The neurons receive inputs from the initial inputs or the interconnections and produce outputs by transformation using an adequate nonlinear transfer function a common transfer function is the sigmoid function expressed by (Tanaka, 2013; Hirose, 2013).

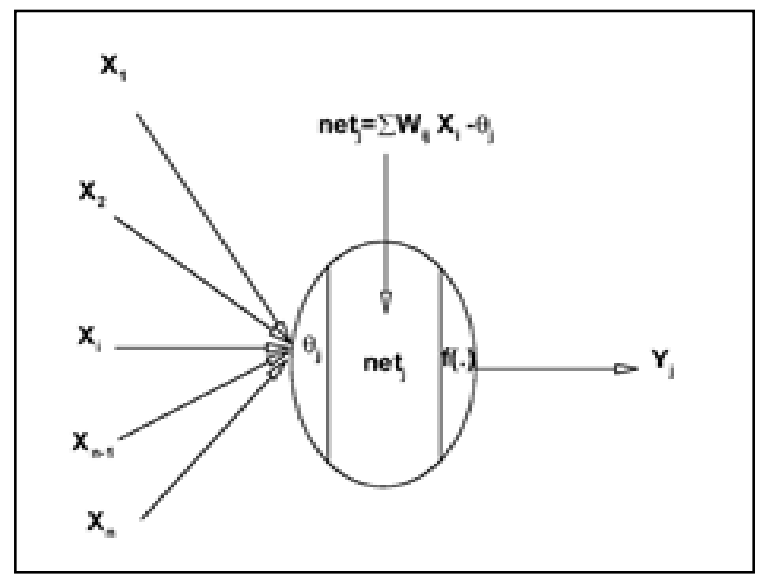

Figure 1 An Artificial Neuron Symbols (T.L. Lee, 2002)

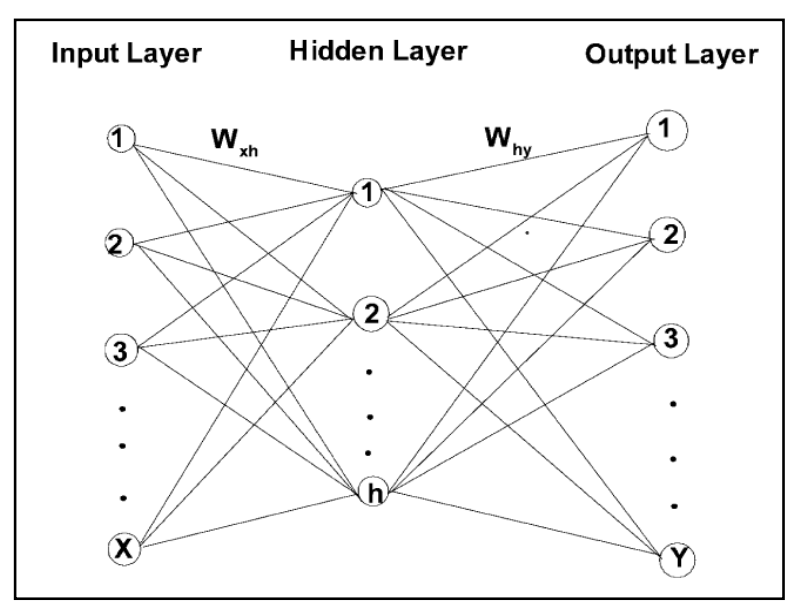

Figure 2 Building of an official Neural Network (T.L. Lee, 2002)

Given a set of input neurons, $X_{i}(I=1,2, \ldots . x)$, the values are multiplied by the first set of interconnection weights, $\left(\mathrm{W}_{\mathrm{xh}}\right)_{\mathrm{ih}}$ where $\left(\mathrm{W}_{\mathrm{xh}}\right)_{\text {ih }}$ is the connection weight from the $\mathrm{i}$-th input neurons to the h-th neurons. The summation of the products, $\mathrm{X}_{\mathrm{i}}\left(\mathrm{W}_{\mathrm{xh}}\right)_{\mathrm{ih}}$, Can be written as

$$
{ }^{n e t}{ }_{h}=\sum_{i} X_{i}\left(W_{x h}\right)_{i h}-\theta_{h h}
$$

Where $\quad \theta_{\mathrm{hh}}$ is the threshold of the h-th hidden neuron.

Each hidden neuron input is then transformed through the transfer function, such as the sigmoid function $f(x)=\left(1+e^{-x}\right)^{-1}$, to produce a hidden neuron output, $\mathrm{H}_{\mathrm{h}}$

$$
H_{h}=f\left(n e t_{h}\right)=\frac{1}{1+e^{-n e t} h}
$$

Similarly, the output value between the hidden layer and the output layer are defined by

$$
\begin{gathered}
n_{j}=\sum_{h} H_{h}\left(W_{h y}\right)_{h j}-\theta_{y j} \\
Y_{j}=f\left(\text { net }_{j}\right)=\frac{1}{1+e^{-n e t} h}
\end{gathered}
$$

In which $\left(\mathrm{W}_{\mathrm{hy}}\right)$ is the connection weight from the h-th hidden neuron to the $\mathrm{j}$-th output neuron, $\theta_{\mathrm{yj}}$ the threshold of the neuron and $Y_{j}$ the value of output layer (T.L. Lee, 2002).

\subsection{The General concept of Wavelet transforms Analysis}

The wavelet transform decomposes signals over dilated and translated functions called wavelets(Bentley and McDonnell, 1994; Kumar and Foufoula-Georgiou, 1994; Walnut, 2013) therefore, the wavelet transforms of a function $f(\mathrm{t})$ with finite energy is defined as the integral transform with a family of functions

$$
\psi_{\lambda, t}(u) \equiv \frac{1}{\sqrt{\lambda}} \psi\left(\frac{u-t}{\lambda}\right)
$$




$$
\begin{aligned}
& W f(\lambda, t)=\int_{-\infty}^{\infty} f(u) \psi_{\lambda, t}(u) d u \\
& \lambda>0 \\
& \quad=\int_{-\infty}^{\infty} f(u) \frac{1}{\sqrt{\lambda}} \psi\left(\frac{u-1}{\lambda}\right) d u
\end{aligned}
$$

$\lambda$ Where $\quad \lambda$ is the scale parameter,
$\mathrm{t}$ is a location parameter and the function

$$
\mathrm{t} \text { is a location parameter }
$$

$$
\psi_{\lambda, u}(u) \text { are called wavelets. }
$$

The necessities of dynamical analysis and the involvement of enormous volume of data to analyze require a technique that can analyze all variables and all frequencies of the tide signal in a steady way, nevertheless, tidal analysis methodology based on continuous wavelet transforms (CWTs) was first proposed by (Flinchem and Jay 2000) and that is able to provide a consistent, linear analysis of tidal and non-tidal variance and reveals features that harmonic analysis is not able to elucidate.

\section{THE MAJOR SHORTCOMINGS OF SOME TIDAL ANALYSIS AND PREDICTION TECHNIQUES}

The requirements of dynamical analysis and the volume of data require that all variables and all frequencies be analysed in a consistent manner, tidal researcher's wish to produce forecasts and hindcasts as well as reconstruct the original data to ameliorate the accuracy of tidal forecasting since early time. Harmonic analysis is termed as the classical model for tidal analysis and prediction, nevertheless, harmonic analysis has its drawback Harmonic analysis method required 18.6 years' time series to resolve all the number of wavelengths of each constituent in the record. Furthermore, classical methods assume that, a given time series is generated from an underlying linear process.

Further problems with the classical harmonic analysis arise in coastal regions where the tidal response is in the form of a wave propagating onshore. Therefore, these methods may not always perform well when applied in modelling hydrological time series. One month of tidal record is approximately required, but harmonic constants cannot be accurately required with harmonic analysis, on the other hand Kalman filtering approach was introduced for short time water level forecasting (Yen et al., 1996). However, the Kalman Filter model is only relevant for short-term prediction, rather than mid-term or long-term prediction. Furthermore, the short-term tidal prediction of sea level requires meteorological data. Therefore, different mathematical techniques were established such as an Artificial Neural Network, Back Propagation Neural Network, Wavelet transformation and hybridization of various models, to overcome the lack of aforementioned observations and to search for an adequate sea level prediction method.

Even though Artificial Neural Network has proven to be a powerful data-driven, self-adaptive, flexible computational tool with the capability of capturing nonlinear and complex underlying characteristics of physical process with a high degree of accuracy it still has its own drawback and the major shortcoming of the neural network methodology is its learning time requirement. Recently Continuous Wavelet Transform (CWT) have been proven to be another satisfactory tool when used to extract harmonic constant without inverse transform by resorting to L1 normalization morlet wavelet transform called inaction method. Inaction method is only appropriate for the short-term tidal level predictions.

The advantage of inaction method over other methods, such as harmonic analysis, fourier transformation is its ability to extract tidal constituent with an instantaneous amplitude, frequency and phase directly via time frequency transform. Another advantage of inaction method is its power to overcome the edge effect for the multi-component signals and obtain the instantaneous frequency, amplitude and phase at the current time. But the major disadvantage of inaction method is it's in ability to differentiate tidal constituent with similar frequencies such as K2 and S2, K1 and P1 when long-term prediction is involved.

This study reviews some selected theoretical perspectives as shown in table 2 in the field of tidal analysis and prediction with a view to provide a broad description of existing approaches. The literature looked into varies tidal analysis modelling categories such as the classical harmonic analysis, Kalman filtering, empirical mode decomposition approach (EMD), normal time frequency transform as it can be seen in Table 2 . However, some of the studies combining two or more types of models such as harmonic analysis and autoregressive integrated moving average-support vector regression, Harmonic analysis with in action method, extreme learning machine and relevance vector machine, harmonic analysis and wavelet network, artificial neural network (ANN) with Kalman filters, back propagation neural network and Cubic B-Spline Curve. But, the analysis shows that classical harmonic analysis remains the most predominant modelling techniques utilized in most of the published work, in spite of the popularity of classical harmonic analysis in tidal prediction its degree of accuracy in some studies is still questionable when short data (one month or a few days) are involved in the analysis and prediction of tide.

The review also revealed that most of the recent tidal analysis approach focused on how to ameliorate the accuracy of prediction via short data as a result of high demand to schedule making on sea surveying such as coastal engineering work, port engineering work, navigation. The idea of applying short data was first initiated by Pei, (1996). The modelling approach shown on the table 2 proved effective in the field of tidal analysis and prediction. However, most of these studies have common limitations. That is, most of them do not predict for mid-term or longer term for example, the most recent work by Song Cai et al., (2018) shows that, his prediction was worse as lead time increase, the method adopted by Son Cai is only appropriate for short-term tidal level predictions and this is common to most of the modelling approaches using short data. Finally, the predictive powers of the aforementioned models shown in table 2 can be greatly improved if further work is done for making mid-term and long term-prediction.

\section{CONCLUSION AND FUTURE DIRECTION}

Tide is the periodic rising and falling of the sea level, and its fluctuations largely influence human lifestyle. Accurate realtime recording of tide level information is essential for ship navigation safety, the development and utilization of marine resources, and marine disaster mitigation and prevention, coastal engineering, oil exploration, etc. Therefore, a simple and efficient tidal prediction method is urgently required. Based on their underlying prediction principles. This paper has 
systematically reviewed publication trends with the current state-of-art for tidal analysis and forecasting technique with the view to identify issues worthy of further investigation, including aspects that have not yet been completely exploited, future research work should pay more attention on long time prediction based on short data since the state of art has sift from single model to integrated approach to ameliorate the accuracy of forecasting via short-time data

\section{ACKNOWLEDGEMENTS}

The authors would like to acknowledge the Federal Government of Nigeria for providing financial support Through Tertiary Education Trust Fund (TETFUND). The author would like to thank the Ministry of Education and Universiti Teknologi Malaysia for funding this research under Research University Grant (Vote number: Q.J130000.2527.12H11).

\section{REFERENCES}

Amiri-Simkooei, A. R., Zaminpardaz, S. and Sharifi, M. A. (2014). 'Extracting tidal frequencies using multivariate harmonic analysis of sea level height time series', Journal of Geodesy, 88(10), pp. 975-988. doi: 10.1007/s00190-014-07375 .

Badejo, O. T. and Akintoye, S. O. (2017). High And Low Water Prediction at Lagos Harbour, Nigeria, Nigerian Journal of Technology. doi: 10.4314/njt.v36i3.39.

Bentley, P. M. and McDonnell, J. T. E. (1994). Wavelet transforms: an introduction, Electronics \& communication engineering journal. IET, 6(4), pp. 175-186.

Bezručka, J. (2011). The Use of a Kalman Filter In Geodesy and Navigation, XIX(2), pp. 8-15. doi: 10.2478/v10189-011-0007y.

Bishop, C. M. (1995). Neural networks for pattern recognition. Oxford university press.

Cai, S., Liu, L. and Wang, G. (2018). Short-term tidal level prediction using normal time-frequency transform, Ocean Engineering. Elsevier, 156, pp. 489-499.

Choi, I. C., Mok, K. M. and Tam, S. C. (2000). Solving harmonic sea-level model with Kalman filter: a Macau case study, Carbonate Beaches, pp. 38-52. doi: $10.1061 / 40640(305) 4$.

Consoli, S., Recupero, D. R. and Zavarella, V. (2014). A survey on tidal analysis and forecasting methods for Tsunami detection, arXiv preprint arXiv:1403.0135.

Deo, M. C. and Chaudhari, G. (1998). Tide Prediction Using Neural Networks, 13, pp. 113-120.

Devlin, A. T. (2016). On the variability of Pacific Ocean tides at seasonal to decadal time scales: Observed vs modelled.

Doodson, A. T. (1954). Appendix to circular-letter 4-H. The harmonic development of the tide-generating potential, International Hydrographic Review, 31, pp. 37-61.
El-Diasty, M. and Al-Harbi, S. (2015). Development of wavelet network model for accurate water levels prediction with meteorological effects, Applied Ocean Research. Elsevier B.V., 53, pp. 228-235. doi: 10.1016/j.apor.2015.09.008.

El-diasty, M., Al-harbi, S. and Pagiatakis, S. (2018). Hybrid harmonic analysis and wavelet network model for sea water level prediction', Physics Procedia. Elsevier B.V., 70, pp. 1421. doi: 10.1016/j.apor.2017.11.007.

Ew, V. O. L. N. O. N. and Ugust, S. E. A. (2003). Prediction and Analysis of Tides and Tidal Currents, Production, 2, pp. 24-29.

Fausett, L. V (1994). Fundamentals of neural networks: architectures, algorithms, and applications. prentice-Hall Englewood Cliffs.

Filippo, A., Torres Jr, A. R., Kjerfve, B., \& Monat, A. (2012). Application of Artificial Neural Network (ANN) to improve forecasting of sea level. Ocean \& coastal management, 55, 101110.

Flinchem, E. P. and Jay, D. A. (2000). An introduction to wavelet transform tidal analysis methods, Estuarine, Coastal and Shelf Science, 51(2), pp. 177-200. doi: 10.1006/ecss.2000.0586.

Foreman, M. G. G., Cherniawsky, J. Y. and Ballantyne, V. A. (2009). Versatile harmonic tidal analysis: Improvements and applications, Journal of Atmospheric and Oceanic Technology, 26(4), pp. 806-817. doi: 10.1175/2008JTECHO615.1.

Foreman, M. G. G. and Henry, R. F. (1989). The harmonic analysis of tidal model time series, Advances in Water Resources, 12(3), pp. 109-120. doi: 10.1016/03091708(89)90017-1.

Godin, G. (1972). The Analysis of Tides. University of Toronto Press, Toronto.

Guo, Z., Pan, H., Cao, A., \& Lv, X. (2018). A harmonic analysis method adapted to capturing slow variations of tidal amplitudes and phases. Continental Shelf Research, 164, 37-44.

Haigh, I. D. (2017). Tides and Water Levels, Encyclopedia of Maritime and Offshore Engineering, pp. 1-13. doi: 10.1002/9781118476406.emoe122.

Hirose, A. (2013). Complex-valued neural networks: Advances and applications. John Wiley \& Sons.

Huang, W., C Murray, N Kraus, and J Rosati (2003).. Development of a regional neural network for coastal water level predictions, Ocean Engineering. Elsevier, 30(17), pp. 2275-2295.

Imani, M., Kao, H. C., Lan, W. H., \& Kuo, C. Y. (2018). Daily sea level prediction at Chiayi coast, Taiwan using extreme learning machine and relevance vector machine. Global and planetary change, 161, 211-221..

Jay, D. A. and Kukulka, T. (2003) Revising the paradigm of tidal analysis-the uses of non-stationary data, Ocean Dynamics. Springer, 53(3), pp. 110-125. 
Karimi, S., Kisi, O., Shiri, J., \& Makarynskyy, O. (2013). Neuro-fuzzy and neural network techniques for forecasting sea level in Darwin Harbor, Australia. Computers \& Geosciences, $52,50-59$

Kumar, P. and Foufoula-Georgiou, E. (1994) Wavelet analysis in geophysics: An introduction, Wavelets in geophysics. Academic Press New York, 4, pp. 1-43.

Lee, T. L. (2004) Back-propagation neural network for longterm tidal predictions, Ocean Engineering, 31(2), pp. 225-238. doi: 10.1016/S0029-8018(03)00115-X.

Lee, W. K. and Lee, W. (2014) Neural Network Approach to Coastal High and Low Water Level Prediction Neural Network Approach to Coastal High and Low Water Level Prediction, (September 2013). doi: 10.1007/978-981-4585-02-6.

Li, S. et al. (2019) Tidal harmonic analysis and prediction with least-squares estimation and inaction method, Estuarine, Coastal and Shelf Science. Elsevier.

Liu, J., Shi, G. and Zhu, K. (2019) High-Precision Combined Tidal Forecasting Model, Algorithms, 12(3), p. 65. doi: 10.3390/a12030065.

Makarynska, D. and Makarynskyy, O. (2008) Predicting sealevel variations at the Cocos (Keeling) Islands with artificial neural networks, Computers and Geosciences, 34(12), pp. 1910-1917. doi: 10.1016/j.cageo.2007.12.004.

Meena, B. L. and Agrawal, J. D. (2015) Tidal level forecasting using ANN, Procedia Engineering. Elsevier B.V., 116(1), pp. 607-614. doi: 10.1016/j.proeng.2015.08.332.

Miller, R. N. and Cane, M. A. (1989) A Kalman filter analysis of sea level height in the tropical Pacific, Journal of Physical Oceanography, 19(6), pp. 773-790.

Mousavian, R. and Hossainali, M. M. (2012) Detection of main tidal frequencies using least squares harmonic estimation method, Journal of Geodetic Science, 2(3), pp. 224-233. doi: 10.2478/v10156-011-0043-6.

Muhammad, A. et al. (2015) Weekly Prediction of tides using Neural networks, Procedia Engineering. Elsevier B.V., 116(Apac), pp. 678-682. doi: 10.1016/j.proeng.2015.08.351.

Najibi, N., Abedini, A. and Sheibani, R. A. (2013) Harmonic Decomposition Tidal Analysis and Prediction Based on Astronomical Arguments and Nodal Corrections in Persian Gulf , Iran, Research Journal of Enviromental and Earth Sciences, 5(7), pp. 381-392.

Okwuashi, O. and Olayinka, D. N. (2017) Tide modelling using the Kalman filter, Journal of Spatial Science. Taylor \& Francis, 62(2), pp. 353-365. doi: 10.1080/14498596.2016.1245162.

Pashova, L. and Popova, S. (2011) Daily sea level forecast at tide gauge Burgas, Bulgaria using artificial neural networks, Journal of Sea Research. Elsevier B.V., 66(2), pp. 154-161. doi: 10.1016/j.seares.2011.05.012.

Pawlowicz, R., Beardsley, B. and Lentz, S. (2002) Classical tidal harmonic analysis including error estimates in Matlab Using T Tide, 28, pp. 929-937.
Pei-Hwa Yen; Chyan-Deng Jan, z Member, ASCE, Youe-Ping Lee, and H.-F. L. (1996) Application Of Kalman Filter To Short-Term Tide Level Prediction, (October), pp. 226-231.

Persson, S. M. and Sharf, I. (2012). Invariant momentumtracking kalman filter for attitude estimation, in 2012 IEEE International Conference on Robotics and Automation. IEEE, pp. 592-598.

Pugh, D. and Woodworth, P. (2014). Sea-level science: understanding tides, surges, tsunamis and mean sea-level changes. Cambridge University Press.

Recupero, D. R. and Zavarella, V. (2013). A Survey On Tidal Analysis And Forecasting Methods For Tsunami Detection', (1), pp. 1-58.

Rose, L. and Bhaskaran, P. K. (2015) Tidal Prediction for Complex Waterways in the Bangladesh Region, Aquatic Procedia, 4(Icwrcoe), pp. 532-539. doi: 10.1016/j.aqpro.2015.02.069.

Rumelhart, D. E., Hinton, G. E. and Williams, R. J. (1988). Learning representations by back-propagating errors, Cognitive modeling, 5(3), p. 1.

Seshadri, V. (2016). Tracking the Variation of Tidal Stature Using Kalman Filter, 2016 International Conference on Circuit, Power and Computing Technologies (ICCPCT). IEEE, pp. 1-6. doi: 10.1109/ICCPCT.2016.7530339.

Shum, C. K., Yu, N. and Morris, C. S. (2001). Recent Advances in Ocean Tidal Science., 測地学会誌. 日本測地学会, 47(1), pp. 528-537.

Slobbe, D. C., Sumihar, J., Frederikse, T., Verlaan, M., Klees, R., Zijl, F., ... \& Broekman, R. (2018). A Kalman filter approach to realize the lowest astronomical tide surface. Marine Geodesy, 41(1), 44-67..

Stephenson, A. (2017). Harmonic Analysis of Tides Using TideHarmonics, 180(1), pp. 1-17.

T.L. Leea, D. S. J. * (2002). Application of artificial neural networks in tide-forecasting, Ocean Engineering, 29(9), pp. 1003-1022. doi: 10.1016/S0029-8018(01)00068-3.

Tanaka, G. (2013). Complex-Valued Neural Networks: Advances and Applications [Book Review], ieee Computational intelligenCe magazine. IEEE, 8(2), pp. 77-79.

Tsai, C.-P. and Lee, T.-L. (1999a). Back-propagation neural network in tidal-level forecasting, Journal of Waterway, Port, Coastal, and Ocean Engineering. American Society of Civil Engineers, 125(4), pp. 195-202.

Tsai, C.-P. and Lee, T.-L. (1999b). Back-Propagation Neural Network in Tidal-Level Forecasting, (August), pp. 195-202.

Vanlede, J. Coen, L. and Deschamps, M. (2014). Tidal Prediction in the Sea Scheldt (Belgium) Using a Combination of Harmonic Tidal Prediction and 1D Hydraulic Modeling, Natural Resources, 05(11), pp. 627-633. doi: 10.4236/nr.2014.511055. 
Walnut, D. F. (2013.) An introduction to wavelet analysis. Springer Science \& Business Media.

Wang, W. and Yuan, H. (2018). A Tidal Level Prediction Approach Based on BP Neural Network and Cubic B-Spline Curve with Knot Insertion Algorithm,

West, B. A., Gagnon, I. F. and Wosnik, M. (2016). Tidal Energy Resource Assessment for McMurdo Station, Antarctica. Engineer Research and Development Center Hanover Nh Hanover United States.

Yen, P. H., Jan, C. D., Lee, Y. P., \& Lee, H. F. (1996). Application of Kalman filter to short-term tide level prediction. Journal of waterway, port, coastal, and ocean engineering, 122(5), 226-231.

Yin, J. C., Wang, N. N. and Hu, J. Q. (2015). A hybrid realtime tidal prediction mechanism based on harmonic method and variable structure neural network, Engineering Applications of Artificial Intelligence. Elsevier, 41, pp. 223-231. doi: 10.1016/j.engappai.2015.03.002.

Zhang, Z., Yin, J., Wang, N., Hu, J., \& Wang, N. (2017). A precise tidal prediction mechanism based on the combination of harmonic analysis and adaptive network-based fuzzy inference system model. Acta Oceanologica Sinica, 36(11), 94-105.

Zhong, C., Jiang, Z., Chu, X., Guo, T., \& Wen, Q. (2019). Water level forecasting using a hybrid algorithm of artificial neural networks and local Kalman filtering. Proceedings of the Institution of Mechanical Engineers, Part M: Journal of Engineering for the Maritime Environment, 233(1), 174-185. 